\title{
Fuzzy Qualitative Simulation With Multivariate Constraints
}

\author{
Wei Pang \\ School of Natural and Computing Sciences \\ University of Aberdeen \\ Aberdeen, UK AB24 3UE \\ Email: pang.wei@abdn.ac.uk
}

\author{
George M. Coghill \\ School of Natural and Computing Sciences \\ University of Aberdeen \\ Aberdeen, UK AB24 3UE \\ Email: g.coghill@abdn.ac.uk
}

\begin{abstract}
In this research we focus on dealing with fuzzy multivariate relations and how we could perform fuzzy qualitative simulation with models containing such relations. To achieve this, we extended Morven, a fuzzy qualitative reasoning framework, and proposed novel types of constraints for the framework. We first introduced fuzzy multivariate function $(F M F)$ constraints, and presented their corresponding constraints in higher differential planes of a Morven model. We then implemented the fuzzy multivariate monotonicity $(F M M)$ relations by $F M F$ constraints and $M M_{-}$add constraints, another kind of constraints we proposed for Morven. In addition, we employed alpha-cut to determine the "strictness" of qualitative signs in the $M M_{-}$add constraints. Finally, proof-of-concept experiments were performed to validate the proposed constraints, and both fuzzy and non-fuzzy situations were considered in these experiments.
\end{abstract}

\section{INTRODUCTION}

Modellers often utilise different model choices when describing complex systems [1]. To describe the dynamic systems with both uncertainty and imprecise properties, fuzzy mechanisms and Qualitative Reasoning (QR) [2], [3] can be combined to form appropriate modelling and simulation tools.

Regarding this FuSim [4], a fuzzy QR system, was developed and it incorporated fuzzy mechanisms into $\mathrm{QR}$, which made it possible to perform fuzzy qualitative simulation. The later fuzzy QR system Morven [5], [6] inherited the fuzzy features of FuSim and introduced several new features compared to its predecessors FuSim and QSIM [7], a nonfuzzy QR system. This makes Morven become the state-ofthe-art fuzzy QR system.

Like QSIM and FuSim, Morven are constraint-based QR systems (CBQR). In CBQR a qualitative model is composed of a set of qualitative constraints, which form a Qualitative Differential Equation (QDE). A QDE model is an abstraction of a set of ODE (Ordinary Differential Equation) models sharing the same structure, and thus able to qualitatively model a complex dynamic system and provide a global picture of the system by capturing qualitative behaviours.

In CBQR systems (both fuzzy and non-fuzzy) qualitative constraints are categorised into two types: algebraic constraints and functional constraints. Algebraic constraints include qualitative addition, subtraction, multiplication, and division, and they are the qualitative versions of the corresponding algebraic operations. Examples of functional constraints are monotonicity constraints, including monotonically increasing and decreasing functions (denoted as $\mathrm{M}^{+}$ and $\mathrm{M}^{-}$) in QSIM, which describe that one variable will monotonically increase with the increase (or decrease) of another.

All algebraic constraints are ternary except qualitative exponentiation. Functional constraints were originally designed to be binary and provided limited capability for reasoning about multivariable functions. Regarding this there was an attempt by Wellman to extend monotonicity constraints in QSIM and make it possible for modelling multivariate relationships [8].

However, Wellman's multivariate constraints were proposed under the QSIM formalism, which are not compatible with later fuzzy CBQR systems FuSim and Morven. This means there is no direct support in Morven for modelling fuzzy multivariate relations. This motivates the research presented in this paper: to develop a scheme for describing fuzzy multivariate relationships in Morven and enable Morven to reason about relations among an arbitrary number of variables, the values of which may be both fuzzy and qualitative.

The rest of the paper is organised as follows: in Section II we introduce the Morven framework. Then we propose the fuzzy multivariate function constraints and fuzzy multivariate monotonicity constraints for Morven in Section III and Section IV, respectively. This is followed by the report of some proof-of-concept experiments in Section V. Finally Section VI concludes the paper and explores some future work.

\section{THE Morven FRAMEWORK}

In this section, we give a detailed description of Morven. In addition, we intend to make the readers familiar with the field of QR by giving concise explanations as well as simple examples. Apart from FuSim's large influence, the development of Morven is also based on Predictive Algorithm (PA) [9] and Vector Envisionment (VE) [10], from which it inherited many features. 


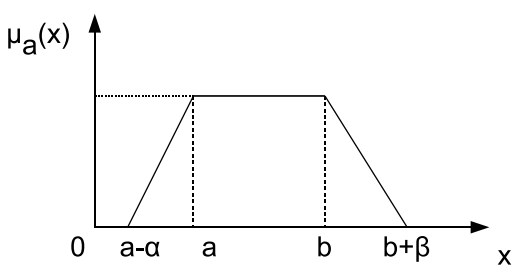

Fig. 1. The Membership Function of a Fuzzy Four-tuple Number

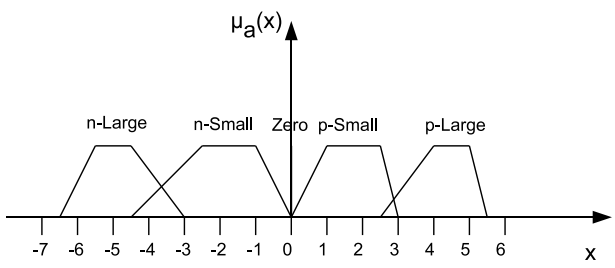

Fig. 2. A Fuzzy Quantity Space

TABlE I. The Signs Quantity Space

\begin{tabular}{c|cccc} 
Quantity & $a$ & $b$ & $\alpha$ & $\beta$ \\
\hline negative(-) & $-\infty$ & 0 & 0 & 0 \\
zero(0) & 0 & 0 & 0 & 0 \\
positive(+) & 0 & $\infty$ & 0 & 0
\end{tabular}

\section{A. Fuzzy Quantity Spaces}

In $\mathrm{QR}$ variables are restricted to take values only from their associated quantity spaces. A quantity space in QSIM is an ordered set of landmark values. In Morven the quantity spaces are extended to the fuzzy domain, and are composed of fuzzy numbers instead of landmark values as in QSIM. For reasons of computational efficiency, trapezoidal fuzzy numbers and intervals are used: each fuzzy number is represented by a fuzzy four tuple $<\mathrm{a}, \mathrm{b}, \alpha, \beta>$, which determines the corresponding membership function, as shown below:

$$
\mu_{A}(x)= \begin{cases}0 & x<a-\alpha \\ \alpha^{-1}(x-a+\alpha) & x \in[a-\alpha, a] \\ 1 & x \in[a, b] \\ \beta^{-1}(b+\beta-x) & x \in[b, b+\beta] \\ 0 & x>b+\beta\end{cases}
$$

Different values of $a, b, \alpha$ and $\beta$ will result in different shapes of membership functions. For instance, the fuzzy number $<\mathrm{a}, \mathrm{a}, 1,1>(\mathrm{a}>0)$ will have a triangle-shape membership function. The general situation is shown in Fig. 1, and one possible fuzzy quantity space is shown in Fig. 2.

The fuzzy quantity space can be considered as a more general representation for quantity spaces. In particular, the signs quantity space, which is often used in many $\mathrm{CBQR}$ systems, can be represented in Table I and illustrated in Fig. 3.

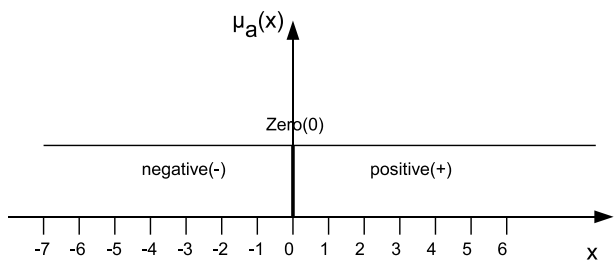

Fig. 3. The Signs Quantity Space

TABLE II. SOME QUALITATIVE CONSTRAINTS IN Morven AND THEIR CORRESPONDING MATHEMATICAL EQUATIONS

\begin{tabular}{ll}
\hline Morven Constraints & Mathematical Equations \\
\hline sub (dt 0 Z, dt 0 X, dt 0 Y) & $Z(t)=X(t)-Y(t)$ \\
add (dt 0 Z, dt 0 X, dt 0 Y) & $Z(t)=X(t)+Y(t)$ \\
mul (dt 0 X, dt 0 Y, dt 0 Z) & $Z(t)=Y(t) * X(t)$ \\
Function (dt 0 Y, dt 0 X) & $Y(t)=f(X(t))$ \\
sub (dt 1 Z, dt 0 X, dt 0 Y) & $d Z(t) / d t=X(t)-Y(t)$ \\
Function (dt 1 Y, dt 0 X) & $d Y(t) / d t=f(X(t))$ \\
\hline
\end{tabular}

\section{B. Fuzzy Qualitative Variables and Constraints}

Variables in Morven are in the form of variable length vectors. The first element in the vector is the magnitude of the variable, and the ith (i>1) element is the (i-1)th derivative. Each element of the variable vector can have its individual fuzzy quantity space.

Algebraic constraints in Morven have the similar form to QSIM and FuSim, but each place in a Morven algebraic constraint is an element of a variable vector, which could be either the magnitude or arbitrary derivative of a variable. Function constraints in Morven are more general than $M^{+}$ and $M^{+}$in QSIM and are many-to-many mappings allowing more flexible descriptions between two variables without knowing the exact mathematical relation.

Table II lists some Morven constraints and their corresponding mathematical equations. In these constraints the label $d t$ means derivative, and the integer immediately following it indicates which derivative of the variable $(0$ means the magnitude). In the right column of the table $X(t)$, $Y(t)$, and $Z(t)$ are continuous functions of time $t$, and $f$ is a function that is continuously differentiable over its domain.

A set of fuzzy arithmetic operations is defined by FuSim [4] and also used by Morven to calculate the algebraic constraints using fuzzy quantity spaces, and details of these operations are given in Table III. These arithmetic operations guarantee that the calculated results are also four-tuple fuzzy numbers. In particular, when a calculated fuzzy number $f_{a}$ is assigned to a variable $V_{a}$, it may not be exactly the same as any available fuzzy number in $V_{a}$ 's associated quantity space $Q$. In this case, the approximation principle [4], [11] will ensure $f_{a}$ can be mapped onto the fuzzy numbers in $Q$, which overlap with it. In addition, the distance metric, which can measure the similarity between two fuzzy numbers, can be used to evaluate the "quality" of the approximation of each mapped fuzzy number in $Q$. The fuzzy number which has the minimum "distance" from $f_{a}$ is the best approximation. 
TABLE III. ARITHMETIC PRIMITIVES USED IN FUSIM AND Morven

\begin{tabular}{lll}
\hline Let: & $m=[a, b, \tau, \beta], n=[c, d, \gamma, \delta]$ & \\
\hline Operation & Result & Conditions \\
\hline$-n$ & $(-d,-c, \delta, \gamma)$ & all $n$ \\
$\frac{1}{n}$ & $\left(\frac{1}{d}, \frac{1}{c}, \frac{\delta}{d(d+\delta)}, \frac{\gamma}{c(c-\gamma)}\right)$ & $n>_{0} 0, n<_{0} 0$ \\
$m+n$ & $(a+c, b+d, \tau+\gamma, \beta+\delta)$ & all $m, n$ \\
$m-n$ & $(a-d, b-c, \tau+\delta, \beta+\gamma)$ & all $m, n$ \\
$m \times n$ & $(a c, b d, a \gamma+c \tau-\tau \gamma, b \delta+d \beta+\beta \delta)$ & $m>_{0} 0, n>_{0} 0$ \\
& $(a d, b c, d \tau-a \delta+\tau \delta,-b \gamma+c \beta-\beta \gamma)$ & $m<_{0} 0, n>_{0} 0$ \\
& $(b c, a d, b \gamma-c \beta+\beta \gamma,-d \tau+a \delta-\tau \delta)$ & $m>_{0} 0, n<_{0} 0$ \\
& $b d, a c,-b \delta-d \beta-\beta \delta,-a \gamma-c \tau+\tau \gamma)$ & $m<_{0} 0, n<_{0} 0$ \\
\hline
\end{tabular}

TABLE IV. MAPpings of A Function Constraint in Morven

\begin{tabular}{l|cccc}
$d t 1 Y \rightarrow d t 0 X$ & n-small & zero & p-small & p-large \\
\hline n-large & 1 & 0 & 0 & 0 \\
n-small & 0 & 1 & 0 & 0 \\
zero & 0 & 0 & 1 & 1 \\
p-small & 0 & 0 & 1 & 1
\end{tabular}

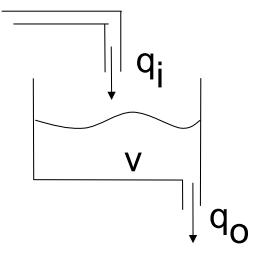

Fig. 4. The Single Tank System

For each Function constraint in Morven a mapping between two variables must be specified. A function constraint Function (V1, V2) (V1, V2 are either the magnitude or arbitrary derivative of a variable) can represent any possible mapping from V1's associated quantity space to V2's. For instance, for the constraint Function (dt 1 Y, dt $0 \mathrm{X}$ ) in Table II, which describes the relation between the first derivative of $\mathrm{Y}$ and the magnitude of $\mathrm{X}$, the mapping could be specified in Table IV, assuming both (dt $1 \mathrm{Y}$ ) and (dt 0 $\mathrm{X}$ )'s quantity spaces are the same as the one shown in Fig. 2. This figure shows a binary relation matrix, in which " 1 " stands for the corresponding mapping is valid. For instance, if the value of ( $\mathrm{dt} 1 \mathrm{Y})$ is zero, then the value of (dt $0 \mathrm{X}$ ) is either $p$-small or $p$-large. Note that the mappings can be many-to-many.

\section{Qualitative Morven Models}

A Morven model is the conjunction of all its qualitative constraints, which are distributed over several differential planes [9]. The Oth differential plane contains the model which is similar to the one used for numerical simulation; constraints in the nth differential planes are obtained by differentiating the corresponding constraints in the $(n-1)$ th differential plane $(n>1)$.

A Morven model for the single tank system shown in Fig. 4 is given in Table V. In this table $V$ is the volume of the liquid in the tank, $q_{i}$ is the inflow, $q_{o}$ is the outflow, and $k$ is a positive constant coefficient. This model is composed of four constraints, $C l$ to $C 4$. The meaning of these constraints has been explained in Section II-B, and the corresponding quantitative relation for each constraint is shown on the right hand side in the brackets. For variable $V$, the magnitude, the
TABLE V. The Morven Model For the Single Tank System

\begin{tabular}{lr}
\hline \hline Differential Plane 0 & \\
\hline C1: Function $\left(\mathrm{dt} 0 q_{o}, \mathrm{dt} 0 \mathrm{~V}\right)$ & $\left(q_{o}=k * V\right)$ \\
C2: sub (dt $\left.1 \mathrm{~V}, \mathrm{dt} 0 q_{i}, \mathrm{dt} 0 q_{o}\right)$ & $\left(V^{\prime}=q_{i}-q_{o}\right)$ \\
\hline Differential Plane 1 & \\
\hline C3: Function (dt $\left.1 q_{o}, \mathrm{dt} 1 \mathrm{~V}\right)$ & $\left(q_{o}^{\prime}=k * V^{\prime}\right)$ \\
C4: sub (dt 2 V, dt1 $\left.q_{i}, \mathrm{dt} 1 q_{o}\right)$ & $\left(V^{\prime \prime}=q_{i}^{\prime}-q_{o}^{\prime}\right)$ \\
\hline \hline
\end{tabular}

TABLE VI. Function MapPings Under the Signs QuANtity SPACE

\begin{tabular}{l|ccc}
\hline \hline Function(A,B) & negative & zero & positive \\
\hline negative & 1 & 0 & 0 \\
zero & 0 & 1 & 0 \\
positive & 0 & 0 & 1 \\
\hline \hline
\end{tabular}

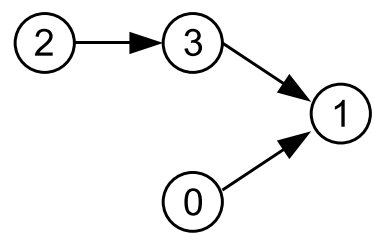

Fig. 5. The Complete Envisionment for the Single Tank System $\left(q_{i}=<\right.$ pos , zer $>$

TABLE VII. The ENVISIONMENT States For the Single TANK SYSTEM

\begin{tabular}{|c|c|c|c|}
\hline State ID & $\overline{\mathrm{V}}$ & $q_{i}$ & $q_{o}$ \\
\hline 0 & $<$ pos , neg, pos $>$ & $<$ pos , zer $>$ & $<$ pos , neg $>$ \\
\hline 1 & $<$ pos, zer, zer $>$ & $<$ pos , zer $>$ & $\langle$ pos , zer $\rangle$ \\
\hline 2 & $<$ zer, pos, neg $>$ & $<$ pos , zer $\rangle$ & $<$ zer, pos $>$ \\
\hline 3 & $<$ pos , pos , neg $>$ & $<$ pos , zer $>$ & $<\operatorname{pos}$, pos $>$ \\
\hline
\end{tabular}

first and second derivatives are used; for variable $q_{o}$ and $q_{i}$, only the magnitude and the first derivative are used.

If all the qualitative variables (including their magnitudes and derivatives) use the signs quantity space, which is shown in Table I, the mappings of the Function in constraint $C l$ and $C 3$ are given in Table VI, in which " 1 " stands for the existence of a mapping between variables $\mathrm{A}$ and $\mathrm{B}$.

\section{Model Output}

After performing qualitative simulation with Morven for the model shown in Table $\mathrm{V}$, the model output could be either an envisionment or a behaviour tree. For example, in the single tank model suppose all the variables take values from the signs quantity space and the input $q_{i}$ remains positive and steady, the simulation results are shown in Fig. 5, in which each node stands for a qualitative state and each directed edge stands for a possible transition between two states.

The corresponding states in this envisionment are listed in Table VII. In this table, pos, zer, and neg stand for positive, zero, and negative, respectively. So the assignment $V=<$ pos, zer, zer $>$ means that the magnitude of $\mathrm{V}$ is positive, the first and second derivatives are zero, and it is similar for the assignments of $q_{i}$ and $q_{o}$. 


\section{FuzZy Multivariate Function CONSTRAints}

From Section II-B we see that function constraints in Morven (also in FuSim) cannot straightforwardly represent multivariate relations. In this section we propose fuzzy multivariate function $(F M F)$ constraints to address this issue. As the Morven framework uses differential planes to hold qualitative constraints, as described in Section II-C, we first give the $F M F$ constraint in the 0th differential plane, and then derive corresponding constraints in higher differential planes.

\section{A. The Fuzzy Multivariate Function (FMF) Constraints in the Oth Differential Plane}

We first naturally extend the function constraints in Morven to be multivariate as follows:

$$
\operatorname{MultiFunction}\left(Y, X \_1, X \_2, \ldots, X \_n\right)
$$

The above $F M F$ constraint will be in the 0 th differential plane and represent a multivariate function:

$$
y(t)=f\left(x_{1}(t), x_{2}(t), \ldots x_{n}(t)\right)
$$

where $n>2$, and $y(t)$ and $x_{i}(t)(1 \leq i \leq n)$ are continuous functions of time $t$ and correspond to $Y$ and $X \_i(1 \leq i \leq$ $n$ ) in Constraint (1), respectively. As Morven variables are in the form of vectors, each place in Constraint (1) are actually the magnitude or derivatives of a variable. For instance, an actual Morven FMF constraint may be as follows:

MultiFunction (dt $1 Y, d t 1 X \_1, d t 0 X \_2, \ldots, d t 0 X \_n$ )

However, for ease of description, we use the variable magnitude form as in Constraint (1). The mapping of the $F M F$ constraints can be obtained from empirical knowledge or observations, similar to the way we specify mappings in Morven function constraints.

\section{B. Corresponding Constraints in the 1st Differential Plane}

Differentiating Equation (2) with respect to time $t$ we obtain the total derivative of $Y(t)$ :

$$
\frac{d y}{d t}=\sum_{i=1}^{n} \frac{\partial y}{\partial x_{i}} \frac{d x_{i}}{d t}
$$

Using Lagrange's notation, Equation (4) can be rewritten as:

$$
y^{\prime}=\sum_{i=1}^{n} f_{x_{i}} x_{i}^{\prime}
$$

where $f_{x_{i}}=\frac{\partial y}{\partial x_{i}}, y^{\prime}=\frac{d y}{d t}$, and $x_{i}^{\prime}=\frac{d x_{i}}{d t}$.

This means in the $1 s t$ differential plane the constraints corresponding to Constraint (1) should be

$$
\begin{gathered}
\text { Function }\left(I t e m \_i, X \_i^{\prime}\right) \\
\operatorname{add}\left(Y^{\prime}, \text { Item_1,Item_2, } \ldots, \text { Item_n }\right)
\end{gathered}
$$

Equations (6) are a set of function constraints $(2 \leq$ $i \leq n)$, and the function mappings of these functions are determined by $f_{x_{i}}$. As algebraic constraints are ternary, Equation (7) is actually a compact representation of the following ternary add constraints:

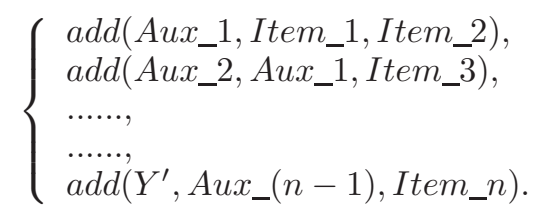

It is noted that in the above add constraints the algebraic operation ("+") is defined in Table III. It is also noted that in the above add constraints Aux_ $i(1 \leq i \leq n-1)$ are auxiliary variables [6], which are used to break long mathematical expressions. Auxiliary variables are used to keep temporary values and will not be mapped back to any quantity spaces. However, the value of $Y$ in the last constraint will be mapped to its associated quantity space by the approximation principle as mentioned in Section II-B.

We can also use one FMF constraint to represent Equation (5):

$$
\text { MultiFunction }\left(Y^{\prime}, X \_1^{\prime}, X \_2^{\prime}, \ldots, X \_n^{\prime}\right)
$$

In the above equation, $Y^{\prime}$ and $X_{-} i^{\prime}(1 \leq i \leq n)$ are derivative of $Y$ and $X \_i$ in Equation (1) with respect to time $t$. The mappings of this $F M F$ constraints is determined by $f_{x_{i}}$.

\section{Corresponding Constraints in the 2nd Differential Plane}

Differentiating Equation (5) with respect to time $t$ will give us the second derivative of $y$ with respect to $t$ :

$$
y^{\prime \prime}=\sum_{i=1}^{n} f_{x_{i}} x_{i}^{\prime \prime}+\sum_{i=1}^{n} \sum_{j=1}^{n}\left(f_{x_{i} x_{j}} x_{i}^{\prime} x_{j}^{\prime}\right)
$$

In the above $y^{\prime \prime}=\frac{d^{2} y}{d t^{2}}, f_{x_{i} x_{j}}=\frac{\partial^{2} f}{\partial x_{i} \partial x_{i}}$. The derivation of Equation (10) is detailed in Appendix A.

From Equation (10) we see that in order to obtain information about the second derivative of $y$, we need to know the Jacobian and Hessian matrices of the multivariate function $f$ in Equation (2). In addition, the second derivative of $y$ is determined by not only $x_{i}^{\prime \prime}$, but also $x_{i}^{\prime}$.

We can use a set of function and $a d d$ constraints to represent Equation (10), similar to the way we use Constraints (6) and (7) to represent Equaiton (5). We assume $n=2$ for ease of description, and Equation (10) becomes:

$$
y^{\prime \prime}=f_{x_{1}} x_{1}^{\prime \prime}+f_{x_{2}} x_{2}^{\prime \prime}+f_{x_{1} x_{1}} x_{1}^{\prime 2}+f_{x_{2} x_{2}} x_{2}^{\prime 2}+2 f_{x_{1} x_{2}} x_{1}^{\prime} x_{2}^{\prime}
$$

The above equation can be represented by the following set of Morven constraints:

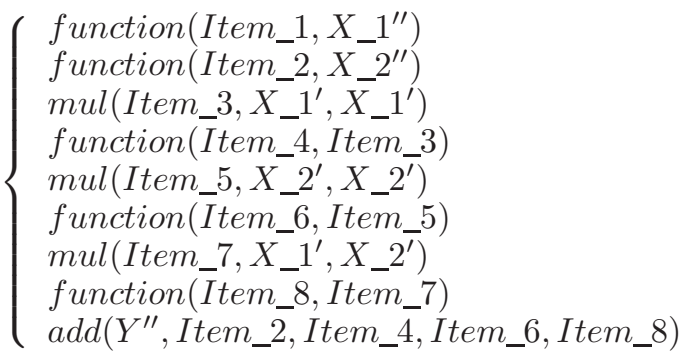


Similar to Equation (9) we can use a single $F M F$ constraint to represent Equaiton (10):

$$
\begin{array}{r}
\operatorname{MultiFunction}\left(Y^{\prime \prime}, X \_1^{\prime}, X \_2^{\prime}, \ldots, X \_n^{\prime},\right. \\
\left.X \_1^{\prime \prime}, X \_2^{\prime \prime}, \ldots, X \_n^{\prime \prime}\right)
\end{array}
$$

In the above $F M F$ constraint the function mappings are determined by the Jacobian $\left(f_{x_{i}}\right)$ and Hessian $\left(f_{x_{i} x_{j}}\right)$ matrices.

\section{FuZZy Multivariate Monotonicity ( $F M M)$ CONSTRAINTS}

In the previous section we gave a general form of $F M F$ constraints and corresponding constraints in higher differential planes. In practice it is sometimes not feasible to use $F M F$ constraints for the following reasons: first, if high resolution fuzzy quantity spaces cannot be associated with derivatives, only signs quantity spaces can be used, as in QSIM. Second, even we can obtain high resolution fuzzy quantity spaces for derivatives, we may not have sufficient knowledge about the underlying multivariate function in terms of its Jacobian and Hessian Matrices.

In the above cases it might still be feasible to study the partial monotonicity of the function, that is, the signs of the Jacobian and Hessian matrices. For instance, we can describe how the resultant variable (i.e., the value of the function) will change with the change of a particular variable in terms of increase and decrease.

\section{A. Wellman's MM Constraints}

Wellman's research on multivariate monotonicity $(M M)$ constraints [8] provides us with meaningful preliminary results. However, Wellman's $M M$ constraints are developed under the QSIM paradigm, and not suitable for the Morven framework: first, it cannot incorporate fuzzy quantity space; second, it assumes the derivatives can only take signs quantity spaces; third, in a Morven model we may need both $M M$ and $F M F$ constraints, because within one model some underlying mulitvariate functions are better understood, which makes it possible to build $F M F$ constraints, and some are not, which requires $M M$ constraints. This situation has to be dealt with.

The above consideration motivates us to develop $M M$ constraints for the Morven framework, or more precisely, fuzzy $M M$ constraints $(F M M)$. The original Wellman's $M M$ constraints are in the following form:

$$
M^{\Delta}\left(x_{1}, \ldots, x_{n}, y\right),
$$

where $\Delta$ is a vector of qualitative signs $\left(\delta_{1}, \delta_{2}, . ., \delta_{n}\right)$ and each $\delta_{i}(1 \leq i \leq n)$ could be,+- , or 0 . Constraint (14) is a natural extension of the binary $M$ constraints in QSIM.

Assuming the underlying function described by Constraint (14) is

$$
y(t)=g\left(x_{1}(t), x_{2}(t), \ldots, x_{n}(t)\right) .
$$

Then the function $g$ will be well constrained by $\Delta$ to be partially monotonic [8], which means the signs of the partial derivative $\frac{\partial y}{\partial x_{i}}$ must be consistent with $\delta_{i}(1 \leq i \leq n)$ in $\Delta$ in Constraint (14). In this paper to be consistent with the representation of Morven FMF constraints, we rewrite Constraint (14) as follows:

$$
M^{\Delta}\left(Y, X_{1}, \ldots, X_{n}\right) .
$$

For instance, when $\Delta=(+,-)$ and $n=2$, the above constraint becomes

$$
M^{(+,-)}\left(Y, X_{1}, X_{2}\right),
$$

which indicates that $\frac{\partial y}{\partial x_{1}}>0$ and $\frac{\partial y}{\partial x_{2}}<0$ in Equation (15). This can be intuitively understood as follows: $y$ will increase (decrease) with the increase (decrease) of $x_{1}$ (if $x_{2}$ remains unchanged), and $y$ will decrease (increase) with the increase (decrease) of $x_{2}$ (if $x_{1}$ remains unchanged). In addition, $y$ will increase (decrease) if $x_{1}$ increases (decreases) and at the same time $x_{2}$ decreases (increases).

\section{B. Implementing Multivariate Monotonicity (MM) Con- straints in Morven}

Recall Sections III-A and III-B, we can use the Morven constraints (including the newly proposed $F M F$ constraints) to implement the $M M$ constraints (this is similar to the way we specialised the Morven function constraints to represent the $M$ constraints in QSIM, details of which are give in [12] and Chapter 4.5 of [13]).

To achieve this we will implement Morven constraints in the Oth and 1st differential planes to represent Constraint (16). In the 0th differential plane we will have an FMF constraint:

$$
\operatorname{MultiFunction}\left(Y, X_{1}, X_{2}, \ldots, X_{n}\right)
$$

The mappings of the above constraint are equivalent to the corresponding values [8] of the $M M$ constraint (16).

In the $1 s t$ differential plane, consider Equation (5) and its Morven implementation Constraints (6) and (7), we give the following abstract constraint to represent partial monotonicity:

$$
M M \_a d d\left(Y^{\prime}, \delta_{1} \operatorname{sgn}\left(X_{1}^{\prime}\right), \delta_{2} \operatorname{sgn}\left(X_{2}^{\prime}\right), \ldots, \delta_{n} \operatorname{sgn}\left(X_{n}^{\prime}\right)\right)
$$

We will explain the above constraint by describing the meaning of each operator. First, $M M \_a d d$ indicates that this is an $a d d$ constraint similar to Constraint (7): the value of $Y^{\prime}$ will be the sum of all the rest of the terms in the constraint. In addition, $M M_{-}$add also means this constraint is a special kind of add constraint used to implement an $M M$ constraint. In this kind of constraints all variables (derivatives) will use the signs quantity space and $\Delta$ in Constraint (16) will be considered. Second, the expression $\operatorname{sgn}(A)$ means we will map the fuzzy four-tuple number that variable (or derivative) $A$ currently takes from its quantity space to the signs quantity space. $\delta_{i}(1 \leq i \leq n)$ is the element of $\Delta$ from Constraint (16), and its value is either + or - (if a $\delta_{i}$ is 0 , the corresponding item $\delta_{i} \operatorname{sgn}\left(X_{i}^{\prime}\right)$ will be 0 , which means this item has no contribution to the value of $Y^{\prime}$ and therefore it can be ignored).

To map the value of variable A to the signs quantity space, we can use alpha-cut to determine how "strict" we want the signs to be. Let the current value of variable A be 
the fuzzy four-tuple number $c=(\mathrm{a}, \mathrm{b}, \alpha, \beta)$, then the signs of A can be determined as follows:

$$
\operatorname{sgn}(A)= \begin{cases}\text { positive }(+) & \text { if }(a-\alpha+\alpha \bar{\alpha} \geq 0) \wedge(b>0) \\ \text { negative }(-) & \text { if }(b+\beta-\beta \bar{\alpha} \leq 0) \wedge(a<0) \\ \text { zero }(0) & \text { if } a=b=\alpha=\beta=0 \\ ? & \text { Otherwise }\end{cases}
$$

In the above, positive, negative, and zero are from the signs quantity space defined in Table I. The question mark "?" means the signs could be,+- , or $0 . \bar{\alpha}$ is the value of alpha-cut, and its range is $[0,1]$. The value of $\bar{\alpha}$ is determined by the domain knowledge and the nature of the associated quantity space of $\mathrm{A}$. if $\bar{\alpha}=0$, we will consider the strictest case and the resultant signs will be the most absolute ones. For instance, the value $c$ is considered to be positive only when its minimum possible value $(a-\alpha)$ is non-negative, and the value of $b$ is positive. Similarly, if $\bar{\alpha}$ is close to 1 , we will have a more relaxed situation.

After calculating $\operatorname{sgn}(A)$, we then consider the items $\delta_{i} \operatorname{sgn}\left(X_{i}^{\prime}\right)$ in Consraint (19). The calculation follows the following rule:

$$
\delta_{i} \operatorname{sgn}\left(X_{i}^{\prime}\right)= \begin{cases}\operatorname{sgn}\left(X_{i}^{\prime}\right) & \text { if } \delta_{i} \text { is }+ \\ -\operatorname{sgn}\left(X_{i}^{\prime}\right) & \text { if } \delta_{i} \text { is - }\end{cases}
$$

Naturally $-\operatorname{sgn}(A)$ is calculated as

$$
-\operatorname{sgn}(A)= \begin{cases}\text { positive } & \text { if } A=\text { negative } \\ \text { zero } & \text { if } A=\text { zero } \\ \text { negative } & \text { if } A=\text { positive }\end{cases}
$$

Having defined all the operations in Constraint (19), we now consider its implementation under the Morven framework.

First we can treat Constraint (19) as an add constraint, and implement it in the same way as we implement Constraint (7) using Constraints (8). However, as we are dealing with signs, it can be simply, and more efficiently, implemented by a set of rules rather than performing the actual fuzzy arithmetic operations. The calculation of $Y^{\prime}$ in Constraint (19) is as follows:

Rules of MM Constraint Checking:

- $\quad Y^{\prime}=+$ iff $\forall i\left(\delta_{i} \operatorname{sgn}\left(X_{i}^{\prime}\right)=0 \vee \delta_{i} \operatorname{sgn}\left(X_{i}^{\prime}\right)=+\right)$ $\wedge \exists i\left(\delta_{i} \operatorname{sgn}\left(X_{i}^{\prime}\right)=+\right)$

- $\quad Y^{\prime}=-\quad$ iff $\forall i\left(\delta_{i} \operatorname{sgn}\left(X_{i}^{\prime}\right)=0 \vee \delta_{i} \operatorname{sgn}\left(X_{i}^{\prime}\right)=-\right)$ $\wedge \exists i\left(\delta_{i} \operatorname{sgn}\left(X_{i}^{\prime}\right)=-\right)$

- $\quad Y^{\prime}=0 \quad$ iff $\forall i\left(\delta_{i} \operatorname{sgn}\left(X_{i}^{\prime}\right)=0\right)$

The above rules can be used to efficiently check whether a qualitative state is consistent with a given Constraint (19). Using these rules, Constraint (19) together with Constraint (18) can represent the same relations described by the $M M$ constraint (16). In this sense we can implement the $M M$ constraints in Morven. In the actual Morven model for the ease of parsing the constraints by the model parser
TABLE VIII. THE Morven Model WITH FMM AND FMF CONSTRAints FOR THE Single TANK System

\begin{tabular}{lr}
\hline \hline Differential Plane 0 & \\
\hline C1: Function $\left(\mathrm{dt} 0 q_{o}, \mathrm{dt} 0 \mathrm{~V}\right)$ & $\left(q_{o}=k * V\right)$ \\
C2: MultiFunction $\left(\mathrm{dt} 1 \mathrm{~V}, \mathrm{dt} 0 q_{i}, \mathrm{dt} 0 q_{o}\right)$ & $\left(V^{\prime}=q_{i}-q_{o}\right)$ \\
\hline Differential Plane 1 & \\
\hline C3: Function $\left(\mathrm{dt} 1 q_{o}, \mathrm{dt} 1 \mathrm{~V}\right)$ & $\left(q_{o}^{\prime}=k * V^{\prime}\right)$ \\
C4: MM_add $(+,-)\left(\mathrm{dt} 2 \mathrm{~V}, \mathrm{dt} 1 q_{i}, \mathrm{dt} 1 q_{o}\right)$ & $\left(V^{\prime \prime}=q_{i}^{\prime}-q_{o}^{\prime}\right)$ \\
\hline \hline
\end{tabular}

TABLE IX. MAPPings of CONSTRAint C2 In TABLE VIII

\begin{tabular}{lll}
\hline dt $1 \mathrm{~V}$ & dt $0 q_{i}$ & dt $0 q_{o}$ \\
\hline positive & positive & zero \\
\hline zero & zero & zero \\
\hline negative & zero & positive \\
\hline
\end{tabular}

and also to make it look similar to an $M M$ constraint, Constraint (19) will be rewritten as follows:

$$
M M \_a d d\left(\delta_{1}, \delta_{2}, \ldots, \delta_{n}\right)\left(Y^{\prime}, X 1^{\prime}, X 2^{\prime}, \ldots, X n^{\prime}\right)
$$

This means when calculating the above constraint within Morven, each $X i^{\prime}$ will be automatically replaced by $\delta_{i} \operatorname{sgn}\left(X_{i}^{\prime}\right)$. An example of Constraint (23) which represents the $M M$ constraint (17) is given as follows:

$$
M M \_a d d(+,-)(d t 1 Y, d t 1 X 1, d t 1 X 2)
$$

It is again noted that in Morven constraints each place is a magnitude or derivative of a variable. So in Constraints (18), (19) and (23) each place is actually the magnitude or derivative of a variable.

\section{PROOF-OF-CONCEPT EXPERIMENTS}

In this section we will perform simulation with the single tank model to demonstrate the validity of $F M F$ and $F M M$ constraints.

\section{A. Single Tank Model}

We first give the single tank model as shown in Table VIII. In this model we assume we are not aware of the algebraic relations among $V^{\prime}, q_{i}$, and $q_{o}$, and instead we use an $M M$ relation represented by one $F M F$ constraint (Constraint $C 2$ ) in the 0 th differential plane and one $M M \_a d d$ constraint (Constraint $\mathrm{C} 4$ ) in the 1 st differential plane.

B. Simulation of Models With Signs Quantity Space and Complete Specification of the Mappings in the FMF Constraint

We first let all variables and derivatives take the signs quantity space, and specify the mappings for Constraint $C 2$ in Table VIII as shown in Table IX.

We then perform both total envisionment (the envisionment that considers all situations of the input $q_{i}$ ) and complete envisionment (the envisionment that assumes certain values of the input variables) when the input $q_{i}$ remains positive and steady. To compare the simulation results we also perform the same total and complete envisionment with the model shown in Table V. Simulation results show that both the model with FMF and FMM constraints and 
TABLE X. Mappings of Constraint $C 2$ In TABle VIII

\begin{tabular}{lll}
\hline $\mathrm{dt} 1 \mathrm{~V}$ & $\mathrm{dt} 0 q_{i}$ & $\mathrm{dt} 0 q_{o}$ \\
\hline positive & positive & zero \\
\hline negative & zero & positive \\
\hline & \\
$\mathrm{V}=<$ zero, zero, zero $>$ \\
$q_{o}=<$ zero, zero $>$ \\
$q_{i}=<$ positive,zero $>$
\end{tabular}

Fig. 6. A Qualitative State of the Single Tank by Morven

the original Morven model give us the same envisionment graphs: for the complete envisionment both models produces the same envisionment graph as shown in Fig. 5; for the total envisionment both models produce 22 qualitative states and 72 possible transitions between these states.

\section{Simulation of Models With Signs Quantity Space and Partial Specification of the Mappings in FMF}

In this section we perform the same total and complete envisionment with the same models as in Section V-B. However, we remove one function mapping of Constraint C2 from Table IX, which gives us the function mappings shown in Table X. This is to demonstrate that we can perform the simulation with only partial specification of the mappings in $F M F$ constraints.

For the complete envisionment, we generate one additional qualitative state compared to the results with complete specification of the mappings, and this state is shown in Figure 6. From this figure we see that because we do not know the algebraic relation among $V^{\prime}, q_{i}$, and $q_{o}$, when $q_{i}=$ positive and $q_{o}=z e r o$, the value of $V^{\prime}$ are assigned to be zero instead of positive. However, the ability to perform this kind of simulation shows that our $F M F$ constraint can deal with incomplete knowledge about the underlying multivariate function and generate results accordingly.

\section{Simulation of Models with Fuzzy Quantity Space}

In this section we perform the simulation with a model of which variables are associated with fuzzy quantity spaces. We first give the following two high resolution fuzzy quantity spaces, as shown in Table XI and Table XII, respectively. We assume that the magnitudes of all variables are associated with Tanks-Quantity-Space-1 in Table XI and the derivatives of all variables are associated with Tanks-Quantity-Space2 in Table XII. The mappings of the function constraint $C 1$ will be both $(d t \quad 0 \quad V)$ and $\left(d t \quad 0 \quad q_{o}\right)$ taking the same values. For instance, if $\left(\begin{array}{lll}d t & 0 & V\end{array}\right)$ is $n$-max, $\left(\begin{array}{lll}d t & 0 & q_{o}\end{array}\right)$ is $n$-max too. Similarly, for Constraint $C 3$ the mappings are also both $(d t \quad 1 \mathrm{~V})$ and $\left(d t \quad 1 q_{o}\right)$ taking the same value: if $(d t \quad 1 V)=p s$-dash, $\left(d t 1 q_{o}\right)$ is $p s$-dash too. The mappings of Constraint $C 2$ is given in Table IX. In addition, we set the value of alpha-cut to be zero.

We then perform the total envisionment, which gives us 257 qualitative states and 2755 transitions. The results are compared with those from the original Morven model shown in Table V, and both models generated the same states and transitions.
TABLE XI. TANKS-QUANTITY-SPACE-1

\begin{tabular}{c|cccc} 
Quantity & $a$ & $b$ & $\alpha$ & $\beta$ \\
\hline n-max & -1 & -1 & 0 & 0.1 \\
n-large & -0.9 & -0.75 & 0.05 & 0.15 \\
n-medium & -0.6 & -0.4 & 0.1 & 0.1 \\
n-small & -0.25 & -0.15 & 0.1 & 0.15 \\
zero & 0 & 0 & 0 & 0 \\
p-small & 0.15 & 0.25 & 0.15 & 0.1 \\
p-medium & 0.4 & 0.6 & 0.1 & 0.1 \\
p-large & 0.75 & 0.9 & 0.15 & 0.05 \\
p-max & 1 & 1 & 0.1 & 0
\end{tabular}

TABLE XII. TANKS-QUANTITY-SPACE-2

\begin{tabular}{c|cccc} 
Quantity & $a$ & $b$ & $\alpha$ & $\beta$ \\
\hline nl-dash & -1 & -0.75 & 0 & 0.15 \\
ns-dash & -0.6 & -0.15 & 0.1 & 0.15 \\
zero & 0 & 0 & 0 & 0 \\
ps-dash & 0.15 & 0.6 & 0.15 & 0.1 \\
pl-dash & 0.75 & 1 & 0.15 & 0
\end{tabular}

\section{E. Remarks}

In this section we have demonstrated the validity of our proposed $F M F$ and $F M M$ constraints by performing fuzzy qualitative simulation with models containing such constraints. We use the single tank system as it is a de facto benchmark system in the field of QR, and it is easier to explain the concepts. We point out that more complex system could have been used, for instance, we have built and simulated more complex models with $F M F$ and $F M M$ constraints based on the minimal interaction model for the osmotic stress response pathway in yeast [14], but using models of such complexity would have made the explanation more tortuous.

In other words, although both $F M F$ and $F M M$ constraints used in the single tank model constraints are ternary, we can easily perform simulation with models containing more complicated $F M F$ and $F M M$ constraints. In this sense the experiments reported in this section are illustrative and proof-of-concept ones.

\section{CONClusions And Future WORK}

In this research we enhance the expressive power of Morven and enable it to deal with fuzzy multivariate relations by introducing two kinds of constraints: multivariate function $(F M F)$ constraints and $M M_{\_}$add constraints. We present the form of $F M F$ constraints across different differential planes, and use $F M F$ and $M M \_a d d$ constraints to describe fuzzy multivariate monotonicity $(F M M)$ relations. This makes it possible to perform fuzzy qualitative simulation with models containing multivariate relations. Proof-of-concept experiments were carried out on the single tank model and simulation results validate the proposed constraints on such benchmark model.

In the future we will conduct theoretical analysis on the completeness and soundness of Morven after introducing multivariate constraints. We will also investigate how the values of alpha-cut in $M M$ add would affect the generation of spurious states. Finally, we are interested in introducing multivariate constraints into QML-Morven [15], a qualitative model learning [16] framework, so that we can automatically learn models containing fuzzy multivariate relations. 


\section{APPENDIX}

\section{A. The Derivation of Equation (10)}

For ease of understanding, we use Leibniz's notion. When differentiating Equation (5) with respect to time $t$, according to the sum rule in differentiation:

$$
\frac{d^{2} y}{d t^{2}}=\frac{d}{d t} \sum_{i=1}^{n}\left(\frac{\partial y}{\partial x_{i}} \frac{d x_{i}}{d t}\right)=\sum_{i=1}^{n} \frac{d}{d t}\left(\frac{\partial y}{\partial x_{i}} \frac{d x_{i}}{d t}\right)
$$

Apply the product rule in differentiation to each item $\frac{d}{d t}\left(\frac{\partial y}{\partial x_{i}} \frac{d x_{i}}{d t}\right)$ of the above equation:

$$
\frac{d}{d t}\left(\frac{\partial y}{\partial x_{i}} \frac{d x_{i}}{d t}\right)=\frac{d}{d t}\left(\frac{\partial y}{\partial x_{i}}\right) \frac{d x_{i}}{d t}+\frac{\partial y}{\partial x_{i}} \frac{d^{2} x_{i}}{d t^{2}}
$$

For the item $\frac{d}{d t}\left(\frac{\partial y}{\partial x_{i}}\right)$ in Equation (26), we express the total derivative of $\frac{\partial y}{\partial x_{i}}$, which is a function of $x_{1}, x_{2}, \ldots, x_{n}$ :

$$
\frac{d}{d t}\left(\frac{\partial y}{\partial x_{i}}\right)=\sum_{j=1}^{n} \frac{\partial}{\partial x_{j}}\left(\frac{\partial y}{\partial x_{i}}\right) \frac{d x_{j}}{d t}=\sum_{j=1}^{n} \frac{\partial y}{\partial x_{j} \partial x_{i}} \frac{d x_{j}}{d t}
$$

Substitute Equation (27) into Equation (26):

$$
\frac{d}{d t}\left(\frac{\partial y}{\partial x_{i}} \frac{d x_{i}}{d t}\right)=\sum_{j=1}^{n} \frac{\partial y}{\partial x_{j} \partial x_{i}} \frac{d x_{j}}{d t} \frac{d x_{i}}{d t}+\frac{\partial y}{\partial x_{i}} \frac{d^{2} x_{i}}{d t^{2}}
$$

Finally substitute Equation (28) into Equation (25):

$$
\frac{d^{2} y}{d t^{2}}=\sum_{i=1}^{n} \sum_{j=1}^{n} \frac{\partial y}{\partial x_{j} \partial x_{i}} \frac{d x_{j}}{d t} \frac{d x_{i}}{d t}+\sum_{i=1}^{n} \frac{\partial y}{\partial x_{i}} \frac{d^{2} x_{i}}{d t^{2}}
$$

Rewrite the above equation using Lagrange's notation, and according to Young's Theorem $f_{x_{i} x_{j}}=f_{x_{j} x_{i}}$, we will obtain Equation (10).

\section{ACKNOWLEDGMENT}

GMC is supported by the CRISP project (Combinatorial Responses In Stress Pathways) funded by the BBSRC (BB/F00513X/1) under the Systems Approaches to Biological Research (SABR) Initiative. WP and GMC are also supported by the partnership fund from dot.rural, RCUK Digital Economy research.

\section{REFERENCES}

[1] R. Leitch, M. Chantler, K. Brown, Q. S. T Kane, G. Coghill, A. S. A. Aldea, J. Liddle, S. McDonnchadha, D. Reay, and M. Ravindranathan, "Modelling choices in intelligent systems," AISB Quarterly, no. 93, pp. 54-60, 1995.

[2] B. Kuipers, Qualitative Reasoning: Modeling and Simulation with Incomplete Knowledge. Cambridge, MA: MIT Press, 1994.

[3] K. D. Forbus, "Qualitative reasoning about physical processes," in IJCAI-81, Vancouver, BC, 1981.

[4] Q. Shen and R. Leitch, "Fuzzy qualitative simulation," IEEE Transactions on Systems, Man, and Cybernetics, vol. 23, no. 4, pp. 10381061, 1993.

[5] G. M. Coghill and M. J. Chantler, "Mycroft: a framework for qualitative reasoning," in Second International Conference on Intelligent Systems Engineering, Hamburg, Germany, September 1994, pp. 4348.
[6] A. M. Bruce and G. M. Coghill, "Parallel fuzzy qualitative reasoning," in Proceedings of the 19th International Workshop on Qualitative Reasoning. Graz, Austria, 2005, pp. 110-116.

[7] B. Kuipers, "Qualitative simulation," Artificial Intelligence, vol. 29 , pp. 289-338, 1986

[8] M. P. Wellman, "Qualitative simulation with multivariate constraints," in Proceedings of the Second International Conference on Principles of Knowledge Representation and Reasoning, 2000, pp. 547-557.

[9] M. Wiegand, "Constructive qualitative simulation of continuous dynamic systems," Ph.D. dissertation, Heriot-Watt university, May 1991.

[10] A. Morgan, "Qualitative behaviour of dynamic physical systems," $\mathrm{Ph} . \mathrm{D}$. dissertation, University of Cambridge, 1988.

[11] G. M. Coghill, "Fuzzy qualitative behaviour prioritisation," in IEEE International Conference on Fuzzy Systems, 2007, pp. 1-6.

[12] W. Pang and G. M. Coghill, "Modified clonal selection algorithm for learning qualitative compartmental models of metabolic systems," in Genetic and Evolutionary Computation Conference (GECCO07), D. Thierens, Ed. New York, NY, USA: ACM Press, 2007, pp. 2887-2894.

[13] W. Pang, "QML-Morven: A framework for learning qualitative models," Ph.D. dissertation, University of Aberdeen, 2009.

[14] J. Macia, S. Regot, T. Peeters, N. Conde, R. Sole, and F. Posas, "Dynamic signaling in the hog1 mapk pathway relies on high basal signal transduction," Science Signaling, vol. 2, no. 63, p. ra13, 2009.

[15] W. Pang and G. M. Coghill, "Qml-morven: A novel framework for learning qualitative models," Department of Computer Science, University of Aberdeen, Tech. Rep. ABDN-CS-12-03, 2012.

[16] _ _ "Learning qualitative differential equation models: a survey of algorithms and applications," The Knowledge Engineering Review, vol. 25 , no. 1 , pp. $69-107,2010$. 